\title{
Network Pharmacology-Based Study on the Molecular Biological Mechanism of Action for Qingdu Decoction against Chronic Liver Injury
}

\author{
Chongyang Ma, ${ }^{1}$ Mengpei Zhao, ${ }^{2}$ Yuqiong Du ${ }^{D},{ }^{1}$ Shuang Jin, ${ }^{3}$ Xiaoyi Wu, ${ }^{1}$ Haiyan Zou, \\ Qiuyun Zhang, ${ }^{1}$ and Lianyin Gao $\mathbb{D}^{1}$ \\ ${ }^{1}$ School of Traditional Chinese Medicine, Capital Medical University, Beijing 10069, China \\ ${ }^{2}$ Kaifeng Second Hospital of Traditional Chinese Medicine, Kaifeng 475000, China \\ ${ }^{3}$ Yanqing County Hospital of Traditional Chinese Medicine, Beijing 102100, China \\ Correspondence should be addressed to Lianyin Gao; 19950032@ccmu.edu.cn
}

Received 24 December 2020; Revised 5 January 2021; Accepted 4 February 2021; Published 4 March 2021

Academic Editor: Chih-Yuan Ko

Copyright ( $\odot 2021$ Chongyang Ma et al. This is an open access article distributed under the Creative Commons Attribution License, which permits unrestricted use, distribution, and reproduction in any medium, provided the original work is properly cited.

Background. Qingdu Decoction (QDD) is a traditional Chinese medicine formula for treating chronic liver injury (CLI). Materials and methods. A network pharmacology combining experimental validation was used to investigate potential mechanisms of QDD against CLI. We firstly screened the bioactive compounds with pharmacology analysis platform of the Chinese medicine system (TCMSP) and gathered the targets of QDD and CLI. Then, we constructed a compound-target network and a protein-protein interaction (PPI) network and enriched core targets in Kyoto Encyclopedia of Genes and Genomes (KEGG) signaling pathways. At last, we used a CLI rat model to confirm the effect and mechanism of QDD against CLI. Enzyme-linked immunosorbent assay (ELISA), western blot (WB), and real-time quantitative polymerase chain reaction (RT-qPCR) were used. Results. 48 bioactive compounds of QDD passed the virtual screening criteria, and 53 overlapping targets were identified as core targets of QDD against CLI. A compound-CLI related target network containing 94 nodes and 263 edges was constructed. KEGG enrichment of core targets contained some pathways related to CLI, such as hepatitis B, tumor necrosis factor (TNF) signaling pathway, apoptosis, hepatitis C, interleukin-17 (IL-17) signaling pathway, and hypoxiainducible factor (HIF)-1 signaling pathway. Three PPI clusters were identified and enriched in hepatitis B and tumor necrosis factor (TNF) signaling pathway, apoptosis and hepatitis B pathway, and peroxisome pathway, respectively. Animal experiment indicated that QDD decreased serum concentrations of alanine aminotransferase (ALT), aspartate aminotransferase (AST), endotoxin (ET), and IL-17 and increased prothrombin time activity (PTA) level. WB and RT-qPCR analyses indicated that, compared with the model group, the expression of cysteinyl aspartate specific proteinase- 9 (caspase-9) protein, caspase-3 protein, B-cell lymphoma-2 associated $X$ protein (Bax) mRNA, and cytochrome c (Cyt c) mRNA was inhibited and the expression of B-cell lymphoma-2 (Bcl-2) mRNA was enhanced in the QDD group. Conclusions. QDD has protective effect against CLI, which may be related to the regulation of hepatocyte apoptosis. This study provides novel insights into exploring potential biological basis and mechanisms of clinically effective formula systematically.

\section{Introduction}

Chronic liver disease (CLD) usually results from iterative liver injury, such as excessive alcohol consumption, viral hepatitis, nonalcoholic fatty liver disease, autoimmune hepatitis, primary biliary cholangitis, and primary sclerosing cholangitis, and causes approximately 2 million deaths per year worldwide [1]. Current treatments for CLD mainly lie in combined medications and artificial liver support, and liver transplant is the only recommended treatment to patients diagnosed with liver failure. Chronic liver injury (CLI), that leads to apoptosis, inflammation, matrix deposition, hyper-bilirubinemia, angiogenesis, and progressive fibrosis, is the hallmark of all types of CLDs, and targeting 
molecular pathways in chronic liver injury would help the development of clinical therapies to prevent or improve the prognosis of CLDs.

Traditional Chinese medicine has been around for thousands of years. There is increasing evidence that certain Chinese medicine prescriptions have beneficial effects on experimental liver injury [2]. Qingdu Decoction (QDD), a traditional Chinese medicine preparation containing Rheum palmatum L., Citrus aurantium L., Magnolia officinalis Rehder and E.H. Wilson, Rehmannia glutinosa (Gaertn.) DC., and Rubia cordifolia L., has notable effects on patients with CLI. Our previous clinical studies have confirmed that the prescription has a good effect on improving clinical symptoms, protecting liver function, and reducing endotoxin absorption in CLI patients $[3,4]$. And our previous experiments have shown that, in TAA-induced model rats, QDD can reduce the level of inflammatory factors and eliminate endotoxin to improve the state of liver damage [5]. We even made some efforts on the mechanisms of QDD against CLI, and a holistic understanding of its molecular mechanisms needs to be further explored.

In the present study, network pharmacology was employed to establish a compound-target network to understand the potential mechanisms of QDD against CLI. The flowchart of the whole study design is illustrated in Figure 1. Firstly, we screened bioactive compounds of QDD and mined their targets using available online databases, and then, targets related to CLI were also obtained. After intersecting these two parts of targets, targets related to both QDD and CLI were obtained and were used for further gene ontology enrichment analysis. Finally, animal experiments were used to confirm the mechanisms predicted through this in silico approach.

\section{Materials and Methods}

2.1. Reagent Supplies. Thioacetamide (TAA) was purchased from Keao Co., Ltd (Beijing, China). Rat ET Enzyme-linked immunoassay (ELISA) kit was from Jiancheng Inst. Biotechnology (Nanjing, China). Rat IL-17 ELISA kit was from eBioscience (Santiago, CA, USA). Primary antibodies used were rabbit caspase- 9 and caspase- 3 antibody (Santa cruz, Dallas, TX, USA) and mouse $\beta$-actin antibody (Zhongshan Jinqiao, Beijing, China). Secondary antibodies used were horseradish peroxidase-conjugated goat anti-rabbit IgG and anti-mouse IgG (Jackson, Lancaster, PA, USA). Total RNA kit was purchased from Tian Gen Biotech (Beijing, China).

2.2. Preparation of QDD. QDD is composed of five Chinese herbs. All the medicinal plants were provided by Beijing Tongrentang Chinese Medicine Co. Ltd. (Beijing, China). Samples were authenticated by a Prof. Zhang Qiuyun from Capital Medical University, China. Every dose of QDD contains Rheum palmatum L. (10 g), Citrus aurantium L. (10 g), Magnolia officinalis Rehder and E.H. Wilson (10 g), Rehmannia glutinosa (Gaertn.) DC. (12 g), and Rubia cordifolia L. $(9 \mathrm{~g})$. The mixture of herbal materials $(51 \mathrm{~g})$ was decocted with distilled water $(2 \times 1 \mathrm{~h}, 1: 10$ for the first extraction and $1: 8$ for the second extraction, w/v). The water extract was filtered and concentrated under reduced pressure to give the aqueous extract of QDD $(34 \mathrm{~mL})$. One milliliter of the extract corresponds to $1.5 \mathrm{~g}$ of crude drug. The decoction was stored at $-20^{\circ} \mathrm{C}$ and heated in a $37^{\circ} \mathrm{C}$ water bath before gavage.

2.3. Animal Model and Treatment. Specific pathogen-free (SPF) grade male Wistar rats (190-210 g) were provided by Charles River Experimental Animals Technology Co. Ltd (Beijing, China). Laboratory animal use and experimental protocols have been reviewed and approved by the Ethics Committee of Capital Medical University (ethics number: AEEI-2017-109). The rats were maintained on a $12 \mathrm{~h}$ light/ dark cycle at constant room temperature $\left(22-25^{\circ} \mathrm{C}\right)$ and humidity (50-70\%), and they have free access to standard rodent food and water.

After 7 days of adaptive feeding, 72 rats were randomly divided into six groups and each group had 12 rats: normal group, model group, lactulose (LA) group, and QDD in a low, moderate, or high dosage (QDDL, QDDM, and QDDH). The normal group was given physiological saline by gavage. The other groups were pretreated with TAA at a dose of $12 \mathrm{mg} / \mathrm{kg}$ for 8 weeks and at an increased dose of $36 \mathrm{mg} / \mathrm{kg}$ from the $9^{\text {th }}$ to the $12^{\text {th }}$ week [6]. In addition, the LA group was fed lactulose solution once a day as a positive control at a dose of $3.5 \mathrm{~mL} / \mathrm{kg}$. Each QDD group was given QDD concentrate (QDDL, QDDM, and QDDH: 5.08, 10.17, and $20.33 \mathrm{~g} / \mathrm{kg}$ ) daily. The whole experiment lasted 12 weeks. After intraperitoneal injection of $3 \%$ sodium pentobarbital anesthetic $(30 \mathrm{mg} / \mathrm{kg})$, all rats were sacrificed by abdominal aorta extraction.

2.4. Serum Biochemical Assay and Plasma Coagulation Analysis. The content of alanine transaminase (ALT) and aspartate transaminase (AST) were measured by automatic biochemical analyzer (Hitachi, Tokyo, Japan). The prothrombin time activity (PTA) was determined with coagulation by an automatic coagulation analyzer (Beckman, LAX, CA, USA).

2.5. Enzyme-Linked Immunoassay (ELISA) Analysis. The content of endotoxin (ET) and IL-17 in serum was measured by multiscan spectrum (Thermo, Waltham, MA, USA) using ET and IL-17 ELISA kits according to the kit's specifications.

2.6. Western Blot Analysis. Total protein was extracted from the liver tissues using cell lysis buffer and analyzed with bicinchoninic acid protein assay kit. Protein samples were separated on sodium dodecyl sulfate (SDS)-polyacrylamide gels and transferred onto a polyvinylidene fluoride membrane. After blocked with 5\% nonfat dry milk in Trisbuffered saline containing 0.05\% Tween-20 (TBST) buffer, membranes were incubated with primary antibody followed by the corresponding horseradish peroxidase-conjugated secondary antibodies. Antibodies and dilutions included the following: rabbit caspase-9 antibody (1:500), mouse 
Qingdu decoction

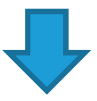

ADME screening

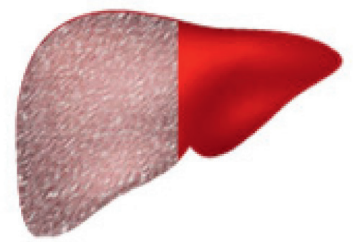

Chronic liver injury
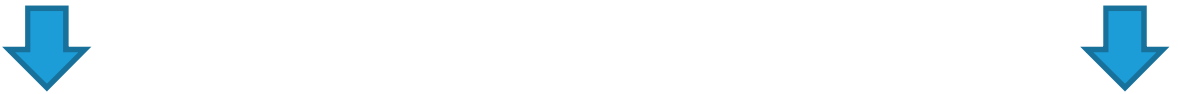

Target collection

Target identification

ᄂ

Intersection

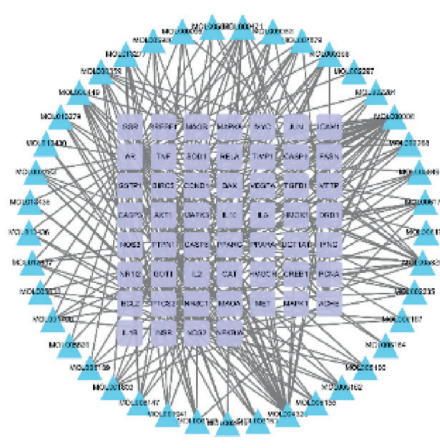

Compound-target network

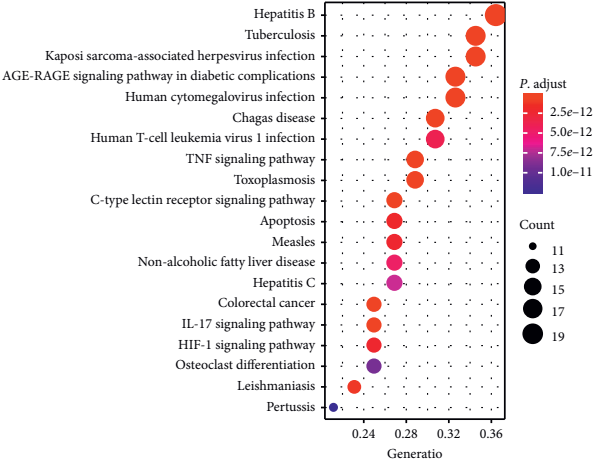

KEGG enrichment

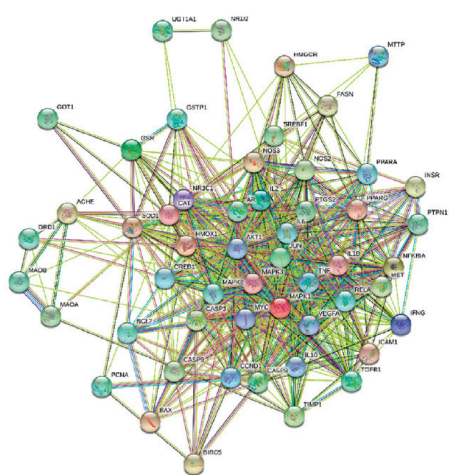

PPI network

Experimental validation

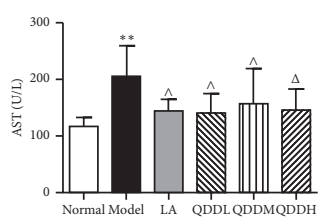

(a)

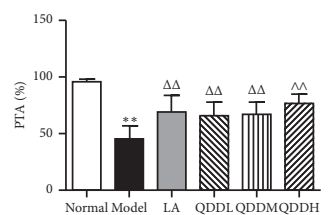

(c)

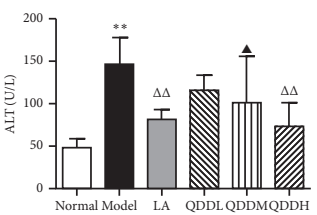

(b)

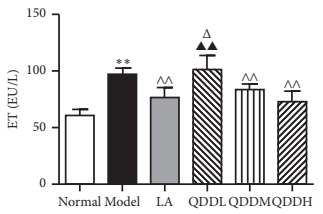

(d)

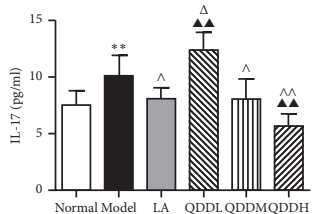

(e)

Anti chronic liver injury

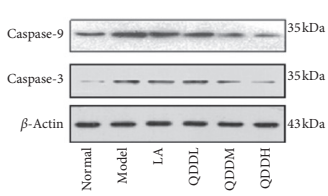

(a)

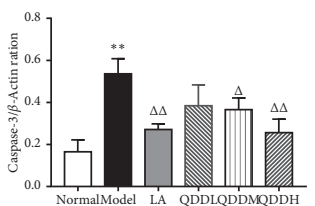

(c)

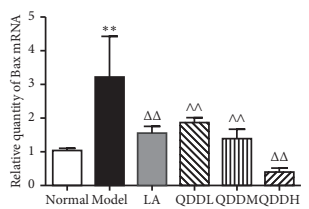

(e)

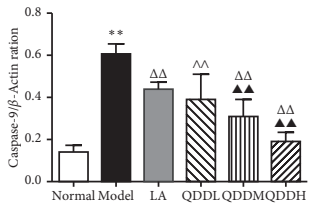

(b)

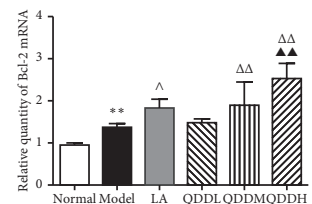

(d)

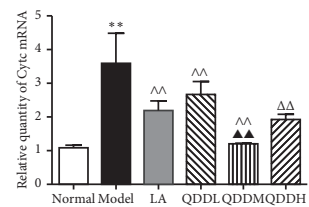

(f)

Figure 1: The flowchart of the whole study design. 
caspase- 3 antibody $(1: 500)$, mouse $\beta$-actin antibody ( 1 : $1,000)$, horseradish peroxidase-conjugated goat anti-rabbit IgG $(1: 10,000)$, and horseradish peroxidase-conjugated goat anti-mouse IgG $(1: 10,000)$.

2.7. Real-Time Quantitative PCR Analysis. Total RNA was extracted from liver tissues using RNA isolation kit according to the manufacturer's specification. Each sample was reverse transcribed into complementary DNA (cDNA) using the HiFi-MMLV cDNA first chain synthesis kit. Gene expression was quantified by means of the comparative $\mathrm{Ct}$ method $(\Delta \Delta \mathrm{Ct})$, and the relative expressions were calculated by the $2^{-\Delta \Delta \mathrm{Ct}}$ method.

2.8. Screening of Active Compounds. All compounds contained in QDD were searched for in the Traditional Chinese Medicine Systems Pharmacology Database and Analysis Platform (TCMSP; http://lsp.nwu.edu.cn/tcmsp.php), containing detailed pharmacological properties of each compound. In the present study, oral bioavailability $(\mathrm{OB})>30 \%$, CACO-2 permeability $>-0.4$, and drug likeness $(\mathrm{DL})>0.18$ were used for bioactive compounds screening for further analyses.

\subsection{Identification of Targets Associated with QDD and CLI.} To predict the target proteins of identified bioactive compounds in QDD, we used an approach integrated with two Chinese medicine databases, including integrative database of traditional Chinese medicine enhanced by symptom mapping (SymMap) and TCMSP [7, 8]. Comparative Toxicogenomics Database (CTD) was used for genes associated with CLI identification [9]. Only genes with Inference Score $>20$ were chosen for further analysis. All these targets were transformed into gene symbols using the UniProt knowledge database (http://www.uniprot.org) with the selected species as Homo sapiens. Subsequently, the intersection of QDD targets and CLI targets was identified as core targets using Draw Venn Diagram online (http://bioinfogp. cnb.csic.es/tools/venny/index.html). Cytoscape 3.7.1 software was used for construction of compound-CLI related target network.

2.10. Construction of PPI Network of Core Targets. The protein-protein interaction (PPI) data of core targets were obtained from STRING database (https://string-db.org/). K-means algorithm was used for PPI cluster identification with default parameters supplied in STRING database. Also, PPI clusters were constructed using Cytoscape 3.7.1 software (National Resource for Network Biology, USA).

2.11. Pathway Enrichment Analysis of Core Targets. To identify the potential biological pathways regulated by QDD against CLI, the ClusterProfiler package of $R$ 3.5.0 was adopted to conduct Kyoto Encyclopedia of Genes and Genomes (KEGG) pathway enrichment analysis of core targets [10]. Adjust $P$ value $<0.05$ was thought to be significant. The top 20 significantly results were further processed to produce a highlevel bubble map and an enrichment map of pathways. KEGG Mapper (https://www.kegg.jp/kegg/mapper.html) was used for pathway visualization.

2.12. Statistical Analysis. All data were presented as means \pm standard error. One-way analysis of variance (ANOVA) was performed using SPSS 19.0 (IBM Corp. Released 2010. IBM SPSS Statistics for Windows, Version 19.0. Armonk, NY, USA) to test the differences between groups. The least-significant difference (LSD) test was used for homogeneity of variance and Tamhane's T2 test for heterogeneity of variance. A value of $P<0.05$ was considered statistically significant.

\section{Results}

3.1. Therapeutic Effect of QDD against CLI. AST and ALT in serum were considered as important indicators of liver injury, and PTA was used to evaluate liver function. As shown in Figures 2(a)-2(c), compared to normal group, AST and ALT levels in the model group were significantly increased $(P<0.01)$, from $115.50 \mathrm{U} / \mathrm{L}$ to $205.30 \mathrm{U} / \mathrm{L}$ and from 47.58 U/L to $145.67 \mathrm{U} / \mathrm{L}$, respectively; and PTA was significantly decreased $(P<0.01)$, from $96.00 \%$ to $45.67 \%$. Compared with the model group, the content of AST in the LA group and each QDD group showed different degrees of reductions $(P<0.05)$, and the content of ALT of LA and QDDH groups were significantly decreased $(P<0.01)$. PTA was significantly increased in each intervention group $(P<0.01)$. Compared with the normal group, endotoxin (ET) and interleukin (IL)-17 levels were increased in the model group $(P<0.01)$. Compared with the model group, the ET and IL-17 levels of the LA group, the QDDM group, and the QDDH group were significantly lower $(P<0.05$ or $P<0.01)$, and these results were shown in Figures $2(d)$ and $2(\mathrm{e})$.

3.2. Screening Bioactive Compounds of QDD. To understand the potential mechanisms of QDD against CLI, a network approach was used as we previous mentioned [11]. After pharmacological properties screening, 48 bioactive compounds were identified for further analysis. As shown in Supplementary Table 1, 9 compounds were found in Dahaung, 19 compounds were found in Zhishi, 2 compounds were found in Houpo, 2 compounds were found in Dihuang, and 18 compounds were found in Qiancao.

3.3. Core Targets of QDD against CLI Using Network Pharmacology Approach. As shown in Supplementary Table 2 and Supplementary Table 3, a total of 813 targets associated with CLI pathogenesis and 172 targets of QDD were obtained from our integrative approach. Furthermore, 53 overlapping targets were identified via mapping targets of QDD into targets related to CLI, which were regarded as the core therapeutic targets of QDD against CLI (Figure 3(a)). Then, a compound-CLI-related target network containing 


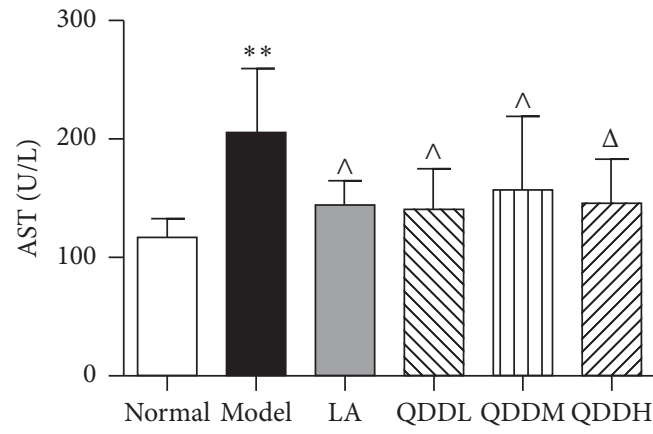

(a)

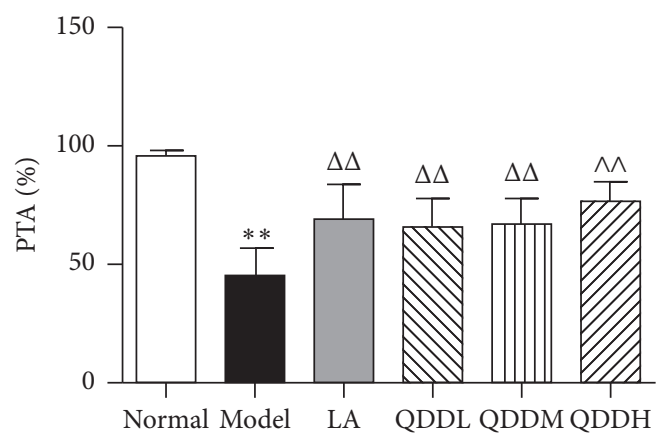

(c)

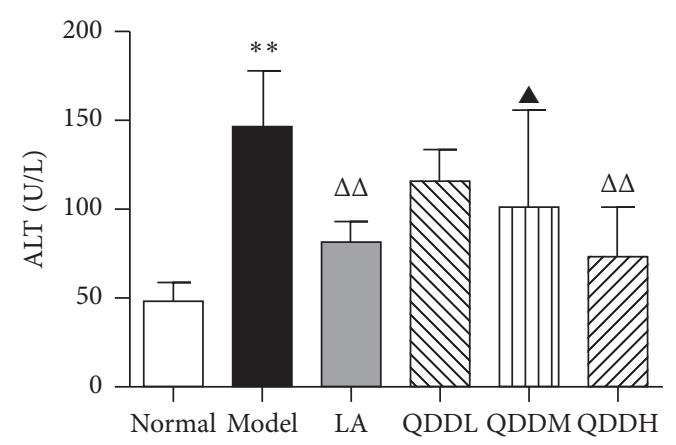

(b)

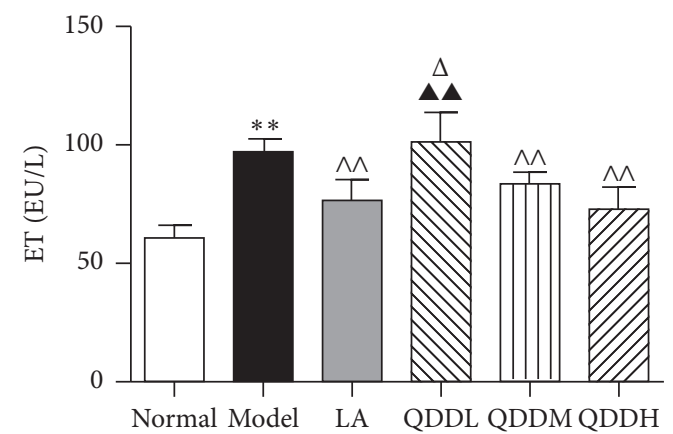

(d)

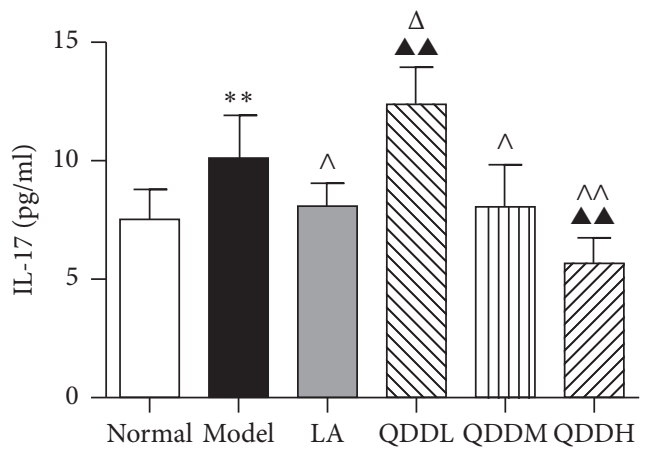

(e)

FIGURE 2: Effects of QDD on blood related indicators: (a) change in serum AST level in each group; (b) change in serum ALT level in each group; (c) change in plasma PTA level in each group; (d) change in serum ET level in each group; (e) change in serum IL-17 level in each group. Values are expressed as mean \pm SEM, $n=6$ for each group, ${ }^{* *} P<0.01$ vs normal group; ${ }^{\Delta} P<0.05$ and ${ }^{\Delta \Delta} P<0.01$ vs model group; $\boldsymbol{\Delta} P<0.05$ and $\boldsymbol{\Delta} \mathbf{\Delta} P<0.01$ vs LA group.

94 nodes and 263 edges was constructed (Figure 3(b)), and top 5 important compounds were identified based on degree values, including luteolin (degree: 27), naringenin (degree: 22), aloe-emodin (degree: 14), nobiletin (degree: 13), and beta-sitosterol (degree: 10).

\subsection{KEGG Pathway Enrichment and PPI Network} Construction. To understand the related signaling pathways of core targets of QDD against CLI, KEGG pathway enrichment analysis was employed via $R$ package. A total of 165 pathways were enriched and top 20 pathways ranked by adjust $P$ value were visualized in a bubble plot (Figure 4(a)). In detail, some pathways related to CLI were obtained, including hepatitis $\mathrm{B}$, TNF signaling pathway, apoptosis, hepatitis C, IL-17 signaling pathway, and hypoxia-inducible factor (HIF-1) signaling pathway. Enrichment map showed that top 20 pathways could be constructed as a network (Figure 4(b)). We focused on the multiple target effect of QDD on apoptosis pathway and visualized these target data in a KEGG diagram. Red nodes represented core targets of QDD against CLI (Figure 4(c)). PPI network of core targets containing 53 nodes and 620 targets was constructed with STRING website (Figure 5(a)). Also, based on topological analysis through K-means algorithm, three PPI clusters were identified (Figures 5(b)-5(d)). After KEGG pathway enrichment, we found that cluster 1 was enriched in the hepatitis B and TNF signaling pathway, cluster 2 was enriched in the apoptosis and hepatitis B pathway, and cluster 3 was enriched in the peroxisome pathway. 


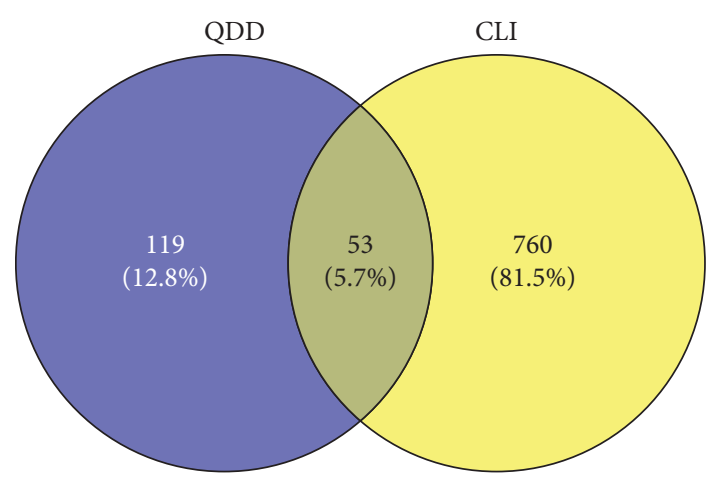

(a)

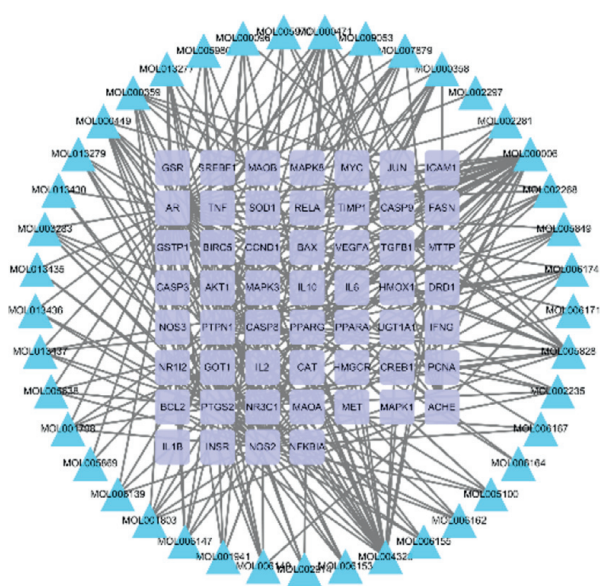

(b)

FIGURE 3: Identification of core targets of Qingdu decoction against chronic liver injury: (a) the Venn diagram of QDD targets and CLI targets; (b) compound-CLI related target network.

3.5. Antiapoptosis Effect of QDD against CLI. The above network pharmacology approach indicated a multiple target effect of QDD on apoptosis pathway, caspase-3, caspase-9, and their upstream signals including Bcl2 and Bax. Western blot analysis showed that the relative expression level of the caspase-9 protein in the model group was significantly increased $(P<0.01)$ compared with the normal group (Figures 6(a) and 6(b)). The relative expression of caspase-9 protein was decreased after treatment with QDD and LA $(P<0.01)$. Compared with the LA group, the relative expression of caspase-9 protein in the QDDM group and the QDDH group was significantly lower $(P<0.01)$. Also, the relative expression level of the caspase- 3 protein in the model group was significantly increased $(P<0.01)$ compared with the normal group (Figures 6(a) and 6(c)). The relative expression of caspase-3 protein was decreased after treatment with middle $(P<0.05)$ and high dose $(P<0.01)$ of QDD. Bcl-2 and Bax are core members of the Bcl-2 family of proteins and play a crucial role in mitochondrial apoptosis [12]. Cyt $c$ is a specific protein that activates caspase-9 [13]. As shown in Figures 6(c) and 6(d), the relative expression levels of Bcl-2, Bax, and Cyt $\mathrm{c}$ genes in the model group were significantly increased by RT-qPCR $(P<0.01)$. Compared with the model group, the relative expression levels of the Bcl-2 gene were increased in all treatment groups $(P<0.05$ or $P<0.01)$ except the QDDL group; the relative expression levels of Bax and Cyt c genes were significantly lower in each treatment group $(P<0.01)$.

\section{Discussion}

Our previously research showed that QDD treatment inhibited CLI induced by TAA and decreased TLR4 signal activation [5]. For QDD was a multiple target preparation, it is necessary to reveal the potential mechanisms in a system level. In the present study, we used a network pharmacology approach to reveal the potential mechanisms of QDD against CLI in a system level. Compound-target network construction indicated that luteolin, naringenin, aloe- emodin, nobiletin, and beta-sitosterol were the most important compounds in the network. Literature evidence supported that all these compounds showed a very good protective effect in various CLI experimental models [14-18]. Evidence supported the ability of aloe-emodin to inhibit diverse events involved in cell apoptosis [19]. Mollugin was a potential nuclear factor kappa-B $(\mathrm{NF} \kappa \mathrm{B})$ inhibitor and decreased expression of inflammatory molecules respond to TNF signal [20, 21]. Rhein was reported to protect liver cells from methotrexate-induced injury through modulating apoptosis-related proteins, such as caspase-3 and $\mathrm{Bcl}-2$ family [22]. On the contrary, some studies reported that all the above compounds could induce apoptosis in many cancer models with different doses [23-25]. Therefore, future studies should explore the differences in drug targets and involve discussion about the relationship between dose and bidirectional effect.

Core targets of QDD against CLI enriched in four categories of pathways, including disease pathways, apoptosis-related pathway, inflammatory pathway, and fibrosisrelated pathway. According to KEGG enrichment analysis, these core targets were also enriched in two diseases pathways, including hepatitis B and hepatitis C. Evidence shows that hepatitis B and hepatitis $\mathrm{C}$ are common causes of CLI, and management of these etiologies leads to a prevention of liver inflammation and fibrosis progression $[26,27]$. Both core targets of QDD against CLI and PPI cluster two were enriched in the apoptosis pathway. According to a reconstructed KEGG map, we found that core targets of QDD against CLI involved in both of the upstream and midstream of apoptosis pathway, such as TNF- $\alpha$, Bcl-2, Bax, and Caspase-9. Our previous study has found that serum level of TNF- $\alpha$ was significantly decreased by QDD in the same CLI model [5]. Therefore, we focused on other targets in this pathway. Besides apoptosis pathway, two inflammatory pathways were obtained, including IL-17 and TNF. TNF pathway activation was the most common phenomenon in kinds of CLI animal models [28-30]. IL-17 is mainly produced from Th17 cells and upregulated in 


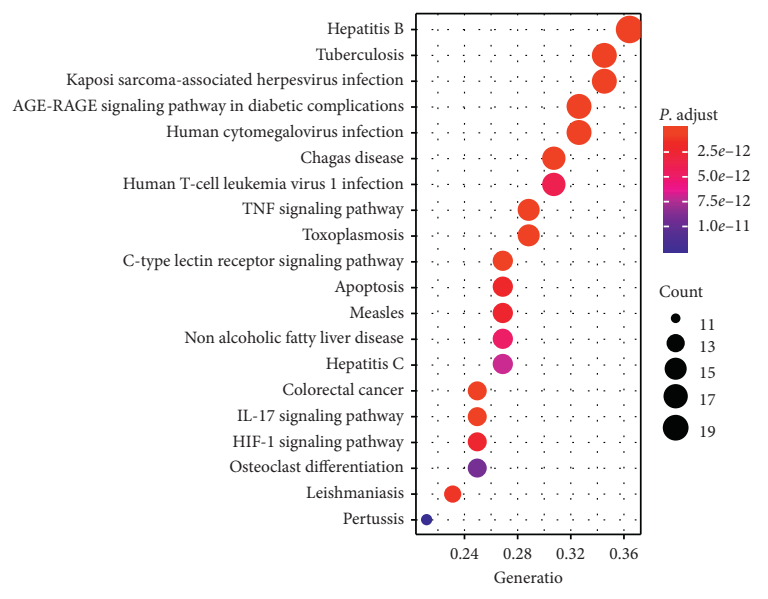

(a)

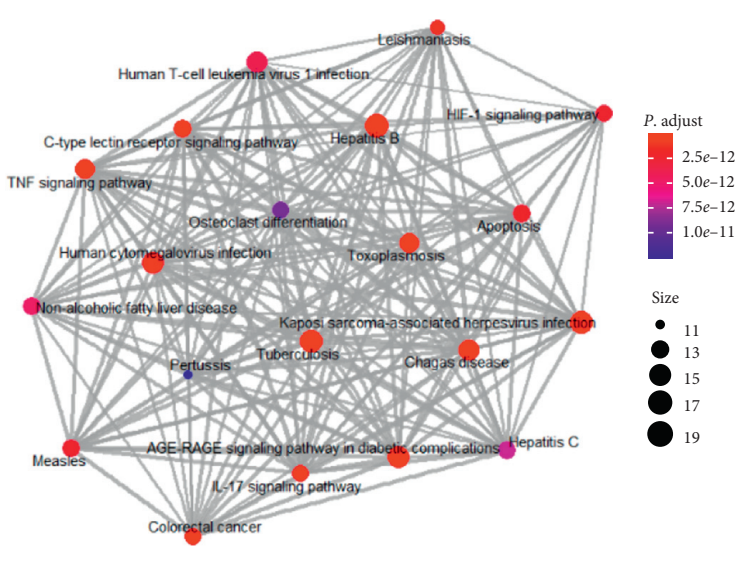

(b)

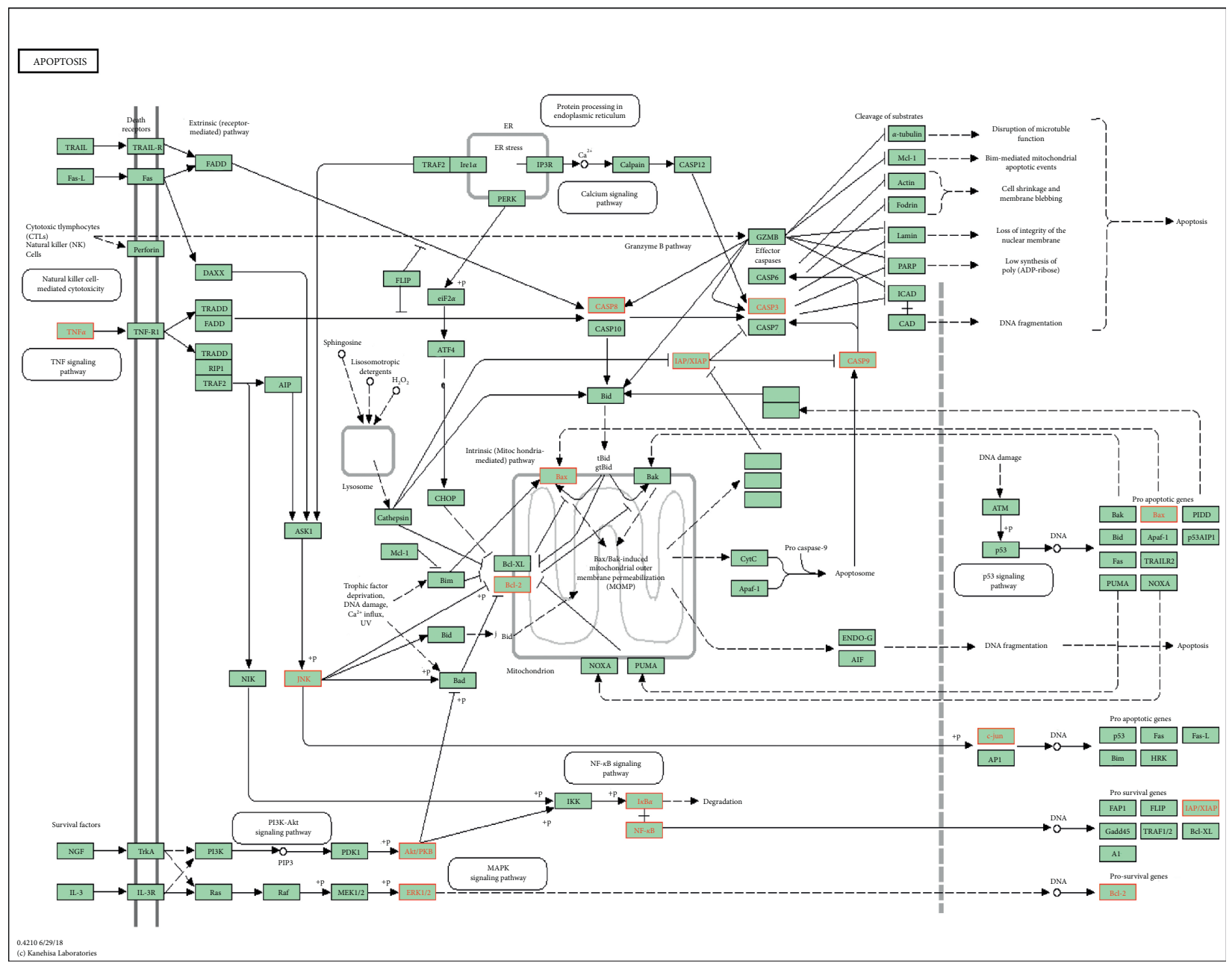

(c)

FIGURE 4: KEGG pathway enrichment analysis of core targets of Qingdu decoction against chronic liver injury: (a) bubble plot of top 20 enriched pathways; (b) enrichment map of top 20 enriched pathways; (c) modulating diagram of QDD on the apoptosis pathway. Red nodes represented core targets of QDD against CLI and green nodes represented proteins in the pathway.

hepatitis B and C, alcoholic liver disease, and autoimmune hepatitis [31]. Literature evidence showed that inhibiting IL17 pathway leaded to a resistance to CLI-induced liver fibrosis [27]. HIF-1 signaling pathway was also identified, and
HIF-1 was confirmed as a critical regulator of profibrotic mediator production during the development of CLI-induced liver fibrosis [32]. The present study focused on the effect of QDD on apoptosis, and we designed a biological 


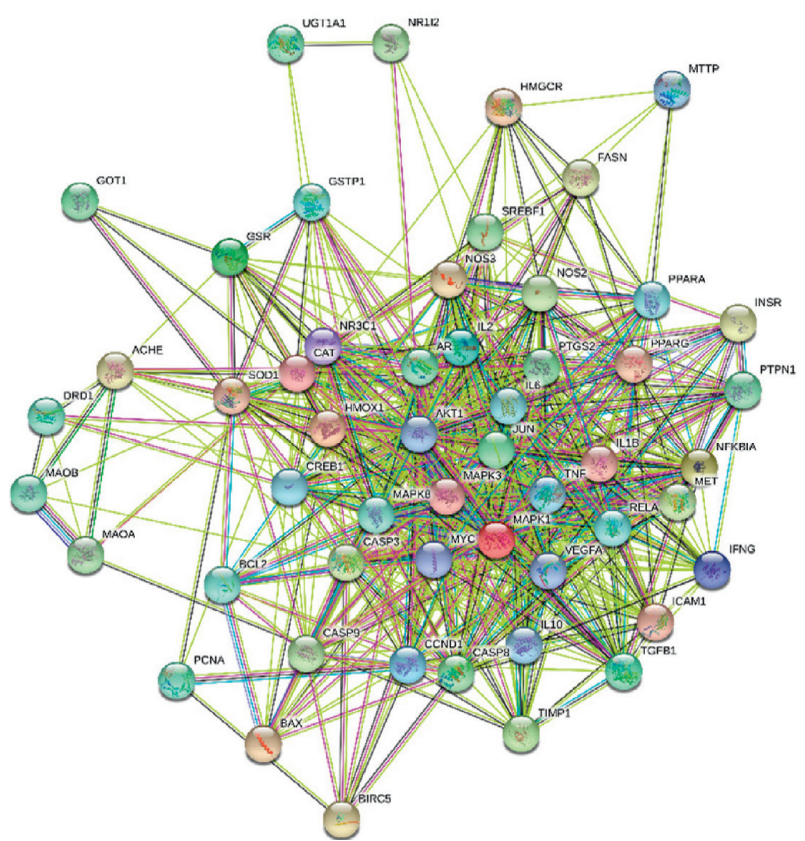

(a)

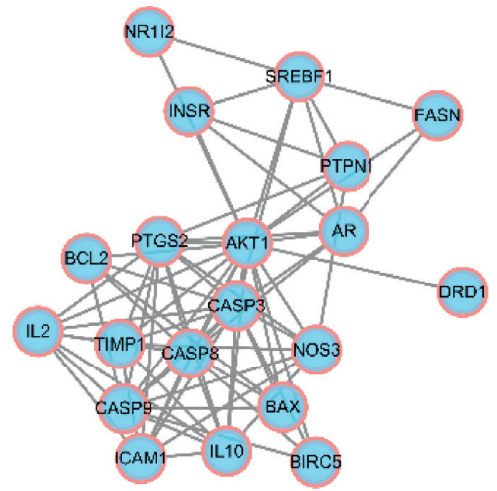

Cluster 2

Apoptosis $(p=4.0 \mathrm{E}-7)$

Hepatitis B $(p=1.2 \mathrm{E}-6)$

(c)

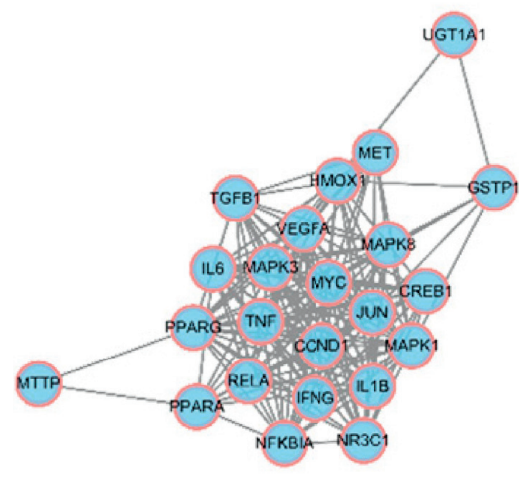

Cluster 1

Hepatitis B $(p=1.4 \mathrm{E}-13)$

TNF signaling pathway $(p=1.6 \mathrm{E}-11)$

(b)

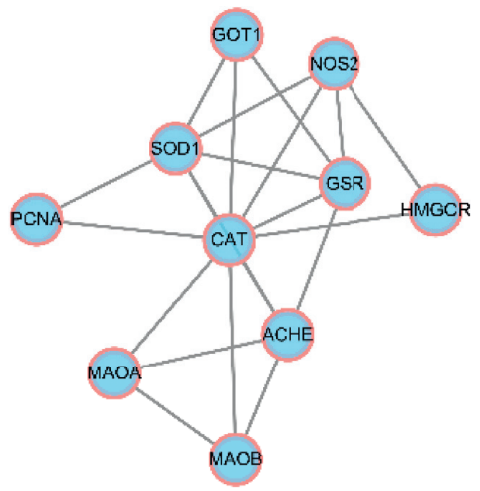

Cluster 3

Peroxisome $(p=4.9 \mathrm{E}-3)$

(d)

FIgURE 5: Protein-protein interaction (PPI) analysis of core targets of Qingdu decoction against chronic liver injury: (a) PPI network of core targets; (b) cluster 1 of the core PPI network; (c) cluster 2 of the core PPI network; (d) cluster 3 of the core PPI network.

molecular experiment to reveal the potential mechanisms of QDD on the apoptosis pathway.

Similar to our previously reported study, we found protective effect of QDD against CLI. AST is mainly distributed in the mitochondria of cells, and ALT is mainly distributed in the cytoplasm of cells [33]. Therefore, the sensitivity of ALT in liver injury is higher than that of AST, and the amplitude of increase is also higher than AST [34]. On the contrary, when AST elevation exceeds ALT, it often indicates that hepatocyte injury is more serious and is a sign of aggravation of chronic disease. Plasma PTA levels mainly reflect the status of liver coagulation function and are also sensitive indicators, reflecting the degree of liver cell damage and prognosis [35].

After confirming the potential anti-CLI effect of QDD, we try to focus on the effects and mechanisms of DQQ against apoptosis. Apoptosis is one of the important causes of liver cell damage, and it is known that endotoxin is the second blow to CLI and induces chronic liver disease progression via gut-liver axis $[36,37]$ and directly damage liver cells and induce hepatocyte apoptosis $[38,39]$. Preclinical evidence showed that LA reduced liver injury and decreased oxidative stress response, excessive inflammatory response, and fibrotic progression via downregulating endotoxin levels [40, 41]. Therefore, LA was used as positive control in the present study. By observing the ultrastructure of hepatocytes and mitochondria, it was found that the mitochondria of hepatocytes in the model group were severely swollen, accompanied by mitochondrial cristae rupture or disappearance. In severe cases, vacuolization or even rupture of the outer membrane was observed. After the 


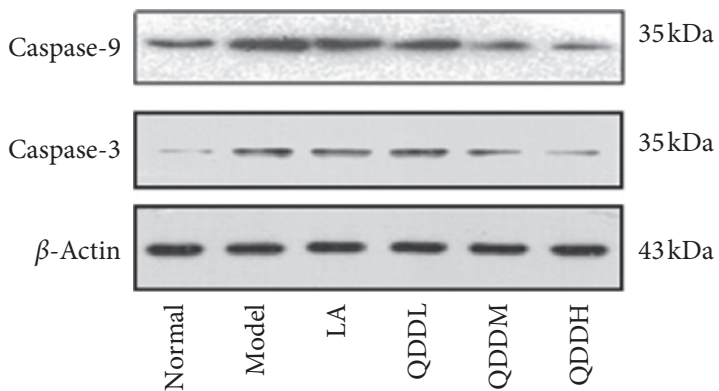

(a)

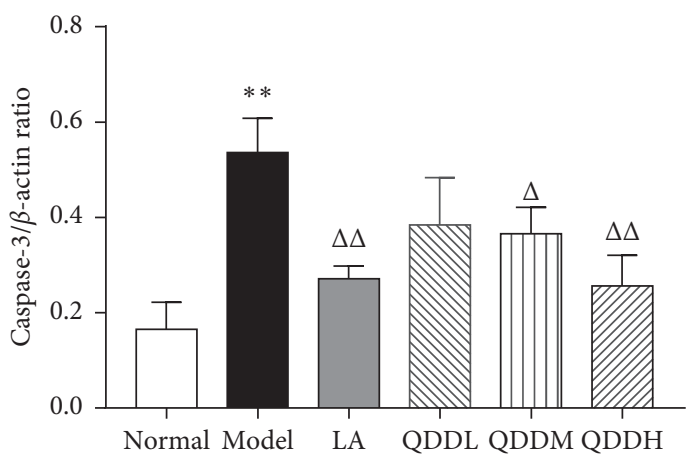

(c)

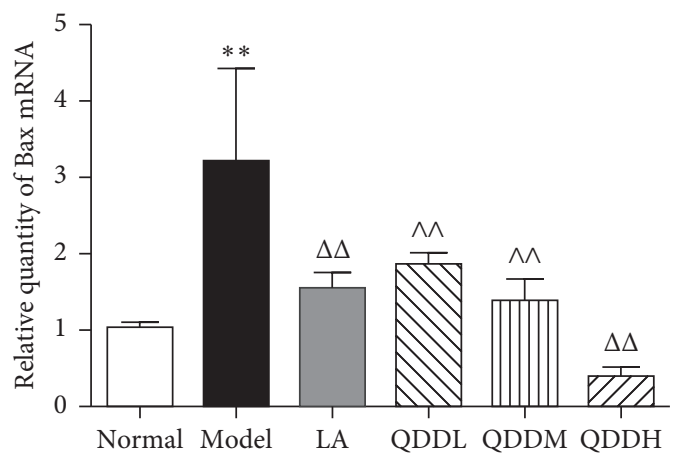

(e)

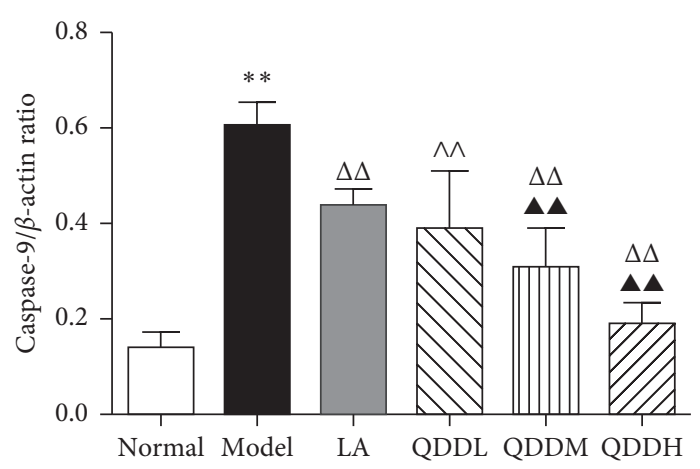

(b)

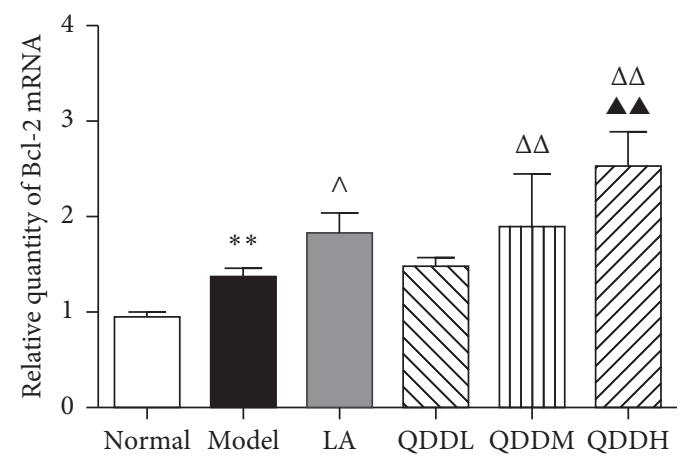

(d)

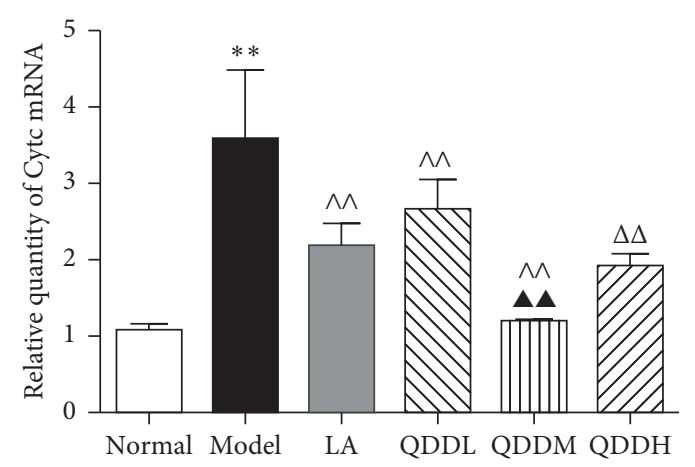

(f)

FIgURE 6: Effects of QDD on hepatocyte apoptosis. (a) Representative results of caspase-9 and caspase-3 protein; (b) the quantitative results of caspase-9 protein; (c) the quantitative results of caspase- 3 protein; (d-f) quantification of Bcl-2, Bax, and Cyt $c$ mRNA. Values are expressed as mean \pm SEM, $n=5$ for each group. ${ }^{* *} P<0.01$ vs normal group; ${ }^{\Delta} P<0.05,{ }^{\Delta \Delta} P<0.01$ vs model group; $\mathbf{\Delta} \mathbf{\Delta} P<0.01$ vs LA group.

intervention of QDD, the apoptosis rate of mitochondria in liver cells was significantly reduced, and the degree of mitochondrial swelling was alleviated. At the same time, it was found that QDD can effectively reduce serum endotoxin level, which was similar to LA treatment.

Inflammatory factor is another inducer of apoptosis activation. Our previous studies confirmed that previously mentioned cytokines via target prediction of QDD, such as TNF- $\alpha$, IL- 6 , and IL- $1 \beta$, were significantly induced by QDD in the same CLI model $[5,42]$. The present study, we tested the serum level of IL-17 following QDD treatment, and the result indicated the potential of IL-17 as a target of QDD. Transcriptomic analysis found that luteolin had a synergistic effect on the expression level of IL-17 pathway-related genes [43], naringenin had a property of T cell-suppressive activity
[44], and nobiletin was also reported to reduce IL-17 level upregulated in inflammatory model [45]. These compounds could be the biological basis of QDD on IL-17 pathway.

Under above stimulating factors, the mitochondrial membrane permeability increased, the membrane potential decreased to gradually disappear, and then, the mitochondrial morphological structure changed, leading to the release of various proapoptotic factors, which in turn leads to apoptosis. Our study found that, during the chronic liver injury, Cyt c was released and activated caspase-9, while Bax expression increased. After QDD intervention, Bax, Cyt c, caspase-9, and caspase-3 were downregulated. The experimental results also showed that the expression of antiapoptosis factor Bcl-2 mRNA was not inhibited, but increased. The role of $\mathrm{Bcl}-2$ family proteins in mitochondrial 
apoptosis is well known [46]. As a core member of Bcl-2 family proteins, the antiapoptotic effect of $\mathrm{Bcl}-2$ is mainly through the stabilization of mitochondrial membrane and regulation of intracellular $\mathrm{Ca}^{2+}$ concentration [47]. Bax, another core member of Bcl-2 family, can promote the release of various proapoptotic factors in mitochondria and can also form specific pores leading to the specific release of Cyt c [48]. Cyt c is a water-soluble protein located in the membrane gap of mitochondria, which is electrostatically stable in physiological conditions and bound to the inner membrane of mitochondria [49]. When stimulated by an apoptosis stimulating factor, Cyt $\mathrm{c}$ is released through the mitochondrial outer membrane, which promotes the binding of caspase- 9 precursor to the amino terminus of apoptotic protease activating factor-1 (Apaf-1) to form an apoptotic body, further activating caspase-9 [50]. Activated caspase- 9 activates the apoptotic executor caspase-3, which ultimately leads to apoptosis [51]. Back to the compoundtarget network, aloe-emodin, naringenin, and sitosterol were found to influence these tested targets directly, future studies should recognize the role of these compound in QDD, and pharmacokinetic parameters should be calculated in both healthy and disease individuals.

\section{Conclusions}

In conclusion, our study demonstrated that QDD can effectively reduce CLI, which is related to regulate hepatocyte apoptosis. The present study provides a new basis for the application of QDD for the treatment of CLI, which is worthy of further study.

$\begin{array}{ll}\text { Abbreviations } \\ \text { QDD: } & \text { Qingdu decoction } \\ \text { LA: } & \text { Lactulose } \\ \text { ALT: } & \text { Alanine aminotransferase } \\ \text { AST: } & \text { Aspartate aminotransferase } \\ \text { ET: } & \text { Endotoxin } \\ \text { IL-17: } & \text { Interleukin-17 } \\ \text { PTA: } & \text { Prothrombin time activity } \\ \text { ELISA: } & \text { Enzyme-linked immunoassay } \\ \text { HE: } & \text { Hematoxylin-eosin } \\ \text { TEM: } & \text { Transmission electron microscopy } \\ \text { WB: } & \text { Western blot } \\ \text { qPCR: } & \text { Quantitative polymerase chain reaction } \\ \text { Bcl-2: } & \text { B-cell lymphoma-2 } \\ \text { Bax: } & \text { Bcl-2-associated } X \text { protein } \\ \text { Cyt c: } & \text { Cytochrome c } \\ \text { Caspase-9: } & \text { Cysteinyl aspartate specific proteinase-9 } \\ \text { TNF- } \alpha: & \text { Tumor necrosis factor- } \alpha \\ \text { IL-6: } & \text { Interleukin-6 } \\ \text { CLI: } & \text { Chronic liver injury. }\end{array}$

\section{Data Availability}

The data used to support the findings of this study are available from the corresponding author upon request.

\section{Ethical Approval}

All experimental animal procedures were performed in strict accordance with Ethics Committee of Capital Medical University.

\section{Consent}

The consent for publication is not applicable.

\section{Conflicts of Interest}

The authors declare that they have no conflicts of interest.

\section{Authors' Contributions}

LG and QZ conceived and designed the study. CM, MZ, YD, SJ, and $\mathrm{HZ}$ performed the study. CM, MZ, YD, SJ, and $\mathrm{HZ}$ analyzed the data. CM, MZ, YD, and XW wrote the paper. LG supported the funding. All authors read and approved the final manuscript. Chongyang $\mathrm{Ma}$ and Mengpei Zhao contributed equally to this work.

\section{Acknowledgments}

The work was supported by the Beijing Municipal Commission of Education (Grant numbers 16ZY01 and KM201810025001).

\section{Supplementary Materials}

Supplementary Table 1. Bioactive compound information of Qingdu Decoction. Supplementary Table 2. Target information of Qingdu Decoction. Supplementary Table 3. Targets associated with chronic liver injury. (Supplementary Materials)

\section{References}

[1] A. M. Moon, A. G. Singal, and E. B. Tapper, "Contemporary epidemiology of chronic liver disease and cirrhosis," Clinical Gastroenterology and Hepatology, vol. 18, no. 12, pp. 26502666, 2020.

[2] J. B. Wang et al., "A systems pharmacology-oriented discovery of a new therapeutic use of the TCM formula Liuweiwuling for liver failure," Scientific Reports, vol. 8, no. 1, p. 5645, 2018.

[3] L. Y. Gao and R. B. Wang, "Qingdu decoction with western medicine treatment of severe hepatitis endotoxemia in 30 cases," Journal of Traditional Chinese Medicine, vol. 44, no. 5, pp. 366-367, 2003.

[4] R. B. Wang, Y. Z. Wu, Y. Y Jiang et al., "Clinical observation of qingdu decoction in the treatment of enterogenous endotoxin in liver disease," Chinese Journal of Integrated Traditional and Western Medicine on Liver Diseases, vol. 14, no. 3, pp. 135137, 2004.

[5] W. Cao, Y Du, L Gao et al., "Lipopolysaccharide/Toll-like receptor 4 signaling pathway involved Qingdu decoction treating severe liver injury merging with endotoxemia," Journal of Traditional Chinese Medicine = Chung I Tsa Chih Ying Wen pan, vol. 37, no. 3, pp. 371-377, 2017.

[6] M. Wallace, K. Hamesch, M. Lunova et al., "Standard operating procedures in experimental liver research: 
thioacetamide model in mice and rats," Laboratory Animals, vol. 49, no. 1_suppl, pp. 21-29, 2015.

[7] C. Lin, F. Wu, T. Zheng, X. Wang, Y. Chen, and X. Wu, "Kaempferol attenuates retinal ganglion cell death by suppressing NLRP1/NLRP3 inflammasomes and caspase- 8 via JNK and NF- $\kappa \mathrm{B}$ pathways in acute glaucoma," Eye, vol. 33, no. 5, p. 777, 2019.

[8] J. Ru, B. Li, P. Li et al., "TCMSP: a database of systems pharmacology for drug discovery from herbal medicines," Journal of Cheminformatics, vol. 6, p. 13, 2014.

[9] A. P. Davis, C. J. Grondin, R. J. Johnson et al., "The comparative toxicogenomics database: update 2019," Nucleic Acids Research, vol. 47, no. D1, pp. D948-d954, 2019.

[10] G. Yu, L.-G. Wang, Y. Han, and Q.-Y. He, "clusterProfiler: an $\mathrm{R}$ package for comparing biological themes among gene clusters," OMICS: A Journal of Integrative Biology, vol. 16, no. 5, pp. 284-287, 2012.

[11] C. Ma, C. Zhai, T. Xuo et al., "A systems pharmacology-based study of the molecular mechanisms of san cao decoction for treating hypertension," Evid Based Complementary Alternative Medicine, vol. 2019, Article ID 3171420, 10 pages, 2019.

[12] A. Panasiuk, J. Dzieciol, B. Panasiuk, and D. Prokopowicz, "Expression of p53, Bax and Bcl-2 proteins in hepatocytes in non-alcoholic fatty liver disease," World Journal of Gastroenterology, vol. 12, no. 38, pp. 6198-6202, 2006.

[13] Y. Hu, M. A. Benedict, L. Ding, and G. Nunez, "Role of cytochrome $\mathrm{c}$ and dATP/ATP hydrolysis in Apaf-1-mediated caspase-9 activation and apoptosis," The EMBO Journal, vol. 18, no. 13, pp. 3586-3595, 1999.

[14] H. Zhang, X. Tan, D. Yang et al., "Dietary luteolin attenuates chronic liver injury induced by mercuric chloride via the Nrf2/NF- $\kappa$ B/P53 signaling pathway in rats," Oncotarget, vol. 8, no. 25, pp. 40982-40993, 2017.

[15] L. Zhao, D. Yan, F. Zhao et al., "Protective effects of five structurally diverse flavonoid subgroups against chronic alcohol-induced hepatic damage in a mouse model," Nutrients, vol. 10, no. 11, 2018.

[16] S. W. Woo, J.-X. Nan, S. H. Lee, E.-J. Park, Y. Z. Zhao, and D. H. Sohn, "Aloe emodin suppresses myofibroblastic differentiation of rat hepatic stellate cells in primary culture," Pharmacology and Toxicology, vol. 90, no. 4, pp. 193-198, 2002.

[17] N. M. Morrow, A. C. Burke, J. P. Samsoondar et al., "The citrus flavonoid nobiletin confers protection from metabolic dysregulation in high-fat-fed mice independent of AMPK," Journal of Lipid Research, vol. 61, no. 3, pp. 387-402, 2020.

[18] E. Devaraj, A. Roy, G. Royapuram Veeraragavan et al., " $\beta$-Sitosterol attenuates carbon tetrachloride-induced oxidative stress and chronic liver injury in rats," NaunynSchmiedeberg's Archives of Pharmacology, vol. 393, no. 6, pp. 1067-1075, 2020.

[19] X. Dong, Y. Zeng, Y. Liu et al., "Aloe-emodin: a review of its pharmacology, toxicity, and pharmacokinetics," Phytotherapy Research, vol. 34, no. 2, pp. 270-281, 2020.

[20] K. J. Kim, J. S. Lee, M. -K. Kwak et al., “Anti-inflammatory action of mollugin and its synthetic derivatives in HT-29 human colonic epithelial cells is mediated through inhibition of NF-kappaB activation," European Journal of Pharmacology, vol. 622 , no. $1-3$, pp. 52-57, 2009.

[21] Z. Wang, M. Y. Li, M. Chunliu, K. S. Wang, J. Ma, and X. Jin, "Mollugin has an anti-cancer therapeutic effect by inhibiting TNF- $\alpha$-induced NF- $\kappa$ B activation," International Journal of Molecular Sciences, vol. 18, no. 8, 1619 pages, 2017.
[22] T. Bu, C. Wang, Q. Meng et al., "Hepatoprotective effect of rhein against methotrexate-induced liver toxicity," European Journal of Pharmacology, vol. 834, pp. 266-273, 2018.

[23] X. Jiang, Y. Ma, M. Ma et al., "Aloe-emodin induces breast tumor cell apoptosis through upregulation of miR-15a/miR16-1 that suppresses BCL2," Evidence Based Complementary Alternative Medicine, vol. 2020, Article ID 5108298, 2020.

[24] L. Zhang, H. Wang, J. Zhu, J. Xu, and K. Ding, "Mollugin induces tumor cell apoptosis and autophagy via the PI3K/ AKT/mTOR/p70S6K and ERK signaling pathways," Biochemical and Biophysical Research Communications, vol. 450, no. 1, pp. 247-254, 2014.

[25] A. KoraMagazi, D. Wang, B. Yousef, M. Guerram, and F. Yu, "Rhein triggers apoptosis via induction of endoplasmic reticulum stress, caspase- 4 and intracellular calcium in primary human hepatic HL-7702 cells," Biochemical and Biophysical Research Communications, vol. 473, no. 1, pp. 230-236, 2016.

[26] G. Sebastiani, K. Gkouvatsos, and K. Pantopoulos, "Chronic hepatitis C and liver fibrosis," World Journal of Gastroenterology, vol. 20, no. 32, pp. 11033-11053, 2014.

[27] E. Seki and D. A. Brenner, "Recent advancement of molecular mechanisms of liver fibrosis," Journal of Hepato-BiliaryPancreatic Sciences, vol. 22, no. 7, pp. 512-518, 2015.

[28] V. Rachakonda, D. Ahmed, N. Shah et al., "M1 muscarinic receptor deficiency attenuates azoxymethane-induced chronic liver injury in mice," Scientific Reports, vol. 5, p. 14110, 2015.

[29] F. J. Cubero, A. Singh, E. Borkham-Kamphorst et al., "TNFR1 determines progression of chronic liver injury in the IKK $\gamma$ / Nemo genetic model," Cell Death and Differentiation, vol. 20, no. 11, pp. 1580-1592, 2013.

[30] B. Knight and G. C. Yeoh, "TNF/LT? double knockout mice display abnormal inflammatory and regenerative responses to acute and chronic liver injury," Cell and Tissue Research, vol. 319, no. 1, pp. 61-70, 2005.

[31] L. Hammerich, F. Heymann, and F. Tacke, "Role of IL-17 and Th17 cells in liver diseases," Clin Dev Immunol, vol. 2011, p. 345803, 2011.

[32] J.-O. Moon, T. P. Welch, F. J. Gonzalez, and B. L. Copple, "Reduced liver fibrosis in hypoxia-inducible factor- $1 \alpha$-deficient mice," American Journal of Physiology-Gastrointestinal and Liver Physiology, vol. 296, no. 3, pp. G582-G592, 2009.

[33] A. Deghady, A. Abdou, W. A. EL-Neanaey, and I. Diab, "Association of genetic polymorphism $-670 \mathrm{~A}>\mathrm{G}$ in the fas gene and serum markers AST platelet ratio index, AST/ALT with significant fibrosis and cirrhosis in chronic hepatitis C," Genetic Testing and Molecular Biomarkers, vol. 16, no. 6, pp. 531-535, 2012.

[34] P. Rief, M. Pichler, R. Raggam et al., “The AST/ALT (De-Ritis) ratio: a novel marker for critical limb ischemia in peripheral arterial occlusive disease patients," Medicine (Baltimore), vol. 95, no. 24, Article ID e3843, 2016.

[35] X. Q. Che, Z. Q Li, Z Chen et al., "Plasma exchange combining with plasma bilirubin adsorption effectively removes toxic substances and improves liver functions of hepatic failure patients," European Review for Medical and Pharmacological Sciences, vol. 22, no. 4, pp. 1118-1125, 2018.

[36] S. De Minicis, C. Rychlicki, L. Agostinelli et al., "Dysbiosis contributes to fibrogenesis in the course of chronic liver injury in mice," Hepatology, vol. 59, no. 5, pp. 1738-1749, 2014.

[37] A. Albillos, A. de Gottardi, and M. Rescigno, "The gut-liver axis in liver disease: pathophysiological basis for therapy," Journal of Hepatology, vol. 72, no. 3, pp. 558-577, 2020. 
[38] M. Xiao, W. Chen, C. Wang et al., "Senescence and cell death in chronic liver injury: roles and mechanisms underlying hepatocarcinogenesis," Oncotarget, vol. 9, no. 9, pp. 8772-8784, 2018.

[39] H. Zhou et al., "Fc-apelin fusion protein attenuates lipopolysaccharide-induced liver injury in mice," Scientific Reports, vol. 8, no. 1, p. 11428, 2018.

[40] B. Taskin, M. A. Erdogan, G. Yiğittürk, D. Günenç, and O. Erbaş, "Antifibrotic effect of lactulose on a methotrexateinduced liver injury model," Gastroenterology Research and Practice, vol. 2017, Article ID 7942531, , 2017.

[41] F. B. Kasravi, D. Adawi, G. Molin, S. Bengmark, and B. Jeppsson, "Effect of oral supplementation of lactobacilli on bacterial translocation in acute liver injury induced by D-galactosamine," Journal of Hepatology, vol. 26, no. 2, pp. 417-424, 1997.

[42] G. L. Luo Jiajia, S. Jin, N. Che, Q. Zhang, Y. Du, and X. Fu, "Effect of Qingdu decoction on intestinal mucosal permeability in rats with severe liver injury," Global Traditional Chinese Medicine, vol. 10, no. 3, pp. 261-264, 2017.

[43] T. J. Lin, Y. Shu-Yi, H. Pei-Wen, and Y. Ning-Sun, I.- Jen Wang, Transcriptomic analysis reveals a controlling mechanism for NLRP3 and IL-17A in dextran sulfate sodium (DSS)induced colitis," Scientific Reports, vol. 8, no. 1, p. 14927, 2018.

[44] X. Niu, C. Wu, M. Li et al., "Naringenin is an inhibitor of T cell effector functions," The Journal of Nutritional Biochemistry, vol. 58, pp. 71-79, 2018.

[45] G. Yang, S. Li, Y. Yang et al., "Nobiletin and 5-Hydroxy-6,7,8,3', $4^{\prime}$ pentamethoxyflavone ameliorate 12-O-Tetradecanoylphorbol-13acetate-Induced psoriasis-like mouse skin lesions by regulating the expression of ki-67 and proliferating cell nuclear antigen and the differentiation of $\mathrm{CD} 4+\mathrm{T}$ cells through mitogen-activated protein kinase signaling pathways," Journal of Agricultural and Food Chemistry, vol. 66, no. 31, pp. 8299-8306, 2018.

[46] R. Wang, H. Zhang, Y. Wang, F. Song, and Y. Yuan, "Inhibitory effects of quercetin on the progression of liver fibrosis through the regulation of NF-KB/IKB $\alpha$, p38 MAPK, and Bcl2/Bax signaling," International Immunopharmacology, vol. 47, pp. 126-133, 2017.

[47] S. Shimamoto, M. Tsuchiya, F. Yamaguchi, Y. Kubota, H. Tokumitsu, and R. Kobayashi, "Ca2+/S100 proteins inhibit the interaction of FKBP38 with Bcl-2 and Hsp90," Biochemical Journal, vol. 458, no. 1, pp. 141-152, 2014.

[48] J. C. Goldstein, N. J. Waterhouse, P. Juin, G. I. Evan, and D. R. Green, "The coordinate release of cytochrome c during apoptosis is rapid, complete and kinetically invariant," Nature Cell Biology, vol. 2, no. 3, pp. 156-162, 2000.

[49] S. Snigdha, E. D. Smith, G. A. Prieto, and C. W. Cotman, "Caspase-3 activation as a bifurcation point between plasticity and cell death," Neuroscience Bulletin, vol. 28, no. 1, pp. 14-24, 2012.

[50] S. B. Bratton and G. S. Salvesen, "Regulation of the apaf-1-caspase9 apoptosome," Journal of Cell Science, vol. 123, no. Pt 19, pp. 3209-3214, 2010.

[51] M. Saikia, R. Jobava, M. Parisien et al., "Angiogenin-cleaved tRNA halves interact with cytochrome c, protecting cells from apoptosis during osmotic stress," Molecular and Cellular Biology, vol. 34, no. 13, pp. 2450-2463, 2014. 